\title{
NEUTRALIZATION OF Bitis parviocula (ETHIOPIAN MOUNTAIN ADDER) VENOM BY THE SOUTH AFRICAN INSTITUTE OF MEDICAL RESEARCH (SAIMR) ANTIVENOM
}

\author{
Elda E. SÁNCHEZ(1), Doug HOTLE(2) \& Alexis RODRÍGUEZ-ACOSTA(3)
}

\begin{abstract}
SUMMARY
Background: The Ethiopian mountain adder (Bitis parviocula) is a viperid known only from a few locations in southwestern Ethiopia. Methods: a total of $30 \mu \mathrm{g}$ of B. arietans and B. parviocula venoms were run on a 10-20\% Tricine gel. To assay lethality dose fifty $\left(\mathrm{LD}_{50}\right)$, five groups of eight mice for each venom were used. Hemorrhagic activity for crude venom was tested. Fibrinogenolytic activity of crude venom was measured using $(2.5 \mathrm{mg} / \mathrm{mL})$ of fibrinogen solution and $(0.03 \mathrm{mg} / \mathrm{mL})$ of crude venom. Gelatinase activity of the venom was tested on a Kodak X-OMAT ${ }^{\mathrm{TM}}$ film. Crude venoms of B. parviocula and B. arietans were tested for their abilities to affect clotting time, clotting rate and platelet function on whole human blood. Results: The (SAIMR) antivenom was confirmed in this study to neutralize the lethal activity of venom from Bitis parviocula. The $\mathrm{ED}_{50} \mathrm{~s}$ of SAIMR antivenom on $B$. parviocula and $B$. arietans neutralized half of 18.2 and $66.7 \mathrm{mg}$ of venom, respectively. The hemorrhagic activities (MHDs) of $B$. parviocula and $B$. arietans were 0.88 and $1.7 \mu \mathrm{g}$, respectively. Bitis arietans and B. parviocula venoms degradated $\alpha$ and $\beta$ chains at different times. The $\gamma$ chains remained unaffected. Bitis parviocula venom did not exhibit gelatinase activity, while $B$. arietans had a MGD of $6.9 \mu \mathrm{g}$. At $3 \mathrm{mg} / \mathrm{mL}$, the crude venoms of B. parviocula and B. arietans did not significantly affect clotting time or clotting rate. Conclusions: The SAIMR antivenom is very effective in neutralizing the venom of $B$. parviocula and should be considered in treating envenomations by these snakes.
\end{abstract}

KEYWORDS: Bitis parviocula; Bitis arietans; South African Vaccine Producers (SAVP); South African Institute of Medical Research (SAIMR) antivenom; Venom.

\section{INTRODUCTION}

The Ethiopian mountain adder (Bitis parviocula) is a medium sized viperid known only from a few locations in southwestern Ethiopia ${ }^{1}$. Until recently only two road-killed specimens and a single live animal had been acknowledged and the species remained in relative scientific obscurity. In 2007, through an agreement with an exporter, twenty of these animals were brought to the United States from Africa ${ }^{10}$. One of the females imported produced young shortly after and this species began to make its way into the hobbyist trade as a high-dollar species.

Given the rarity of this snake, little is known about the composition or toxicity of the venom ${ }^{8,14}$. Indigenous people of southern Ethiopia consider this snake highly dangerous ${ }^{14}$. Epidemiology is virtually nonexistent although given the human population in southern Ethiopia; it is thought to inflict a reasonable number of bites each year ${ }^{14}$. The venom has traditionally been presumed to be cytotoxic similar to congenerics. Some cross-reactivity with polyvalent antivenoms produced against Bitis was suspected ${ }^{4}$. In 2009, an amateur herpetologist in San Antonio, Texas was envenomated by a young $B$. parviocula while attempting to ready the animal for shipment. Attending physicians began to administer the South African polyvalent antivenom SAIMR (South African Institute of Medical Research). However, after $3 / 4$ of the initial vial was given the patient showed signs of anaphylaxis and the antivenom was stopped at that point. Given the negligible dose, it remained unclear if the SAIMR would be an effective treatment for this species ${ }^{5,8}$. In this study, SAIMR was tested for its neutralizing ability on the lethal toxicity of B. pariviocula venom. The lethal and proteolytic activities of this venom were also compared to those of the closely related African Puff adder (Bitis arietans) ${ }^{6}$.

\section{MATERIALS AND METHODS}

Snake collection and husbandry: The $B$. parviocula were collected in SE Ethiopia, Africa (8²7' S 1.54' N 36 21' 4.99'E; Fig. 1) and shipped to a private individual in the United States. These animals were housed in the private collection of $\mathrm{Al}$ Coritz. The snakes were held in Vision ${ }^{\circledR}$ snake enclosures and kept at $18-25^{\circ} \mathrm{C}$. White laboratory mice (Mus musculus) were offered as food once every seven days. Fresh water was available ad libitum. B. arietans used in this study were collected between Arusha and Dar es Salaam, Tanzania, Africa. (5º2' 54.91S $37^{\circ} 3.74684$ E; Fig. 1).

The authors affirm that this work has never been presented in any scientific reunion and these data has never been published elsewhere.

(1) National Natural Toxins Research Center, Texas A\&M University-Kingsville and the Department of Chemistry, Texas A\&M University-Kingsville, Kingsville, TX, USA.

(2) Albuquerque Biological Park, Albuquerque, NM.

(3) Immunochemistry Section, Tropical Medicine Institute and Instituto Nacional de Higiene "Rafael Rangel", Caracas Venezuela.

Correspondence to: Elda E. Sánchez. E-mail: elda.sanchez@tamuk.edu 


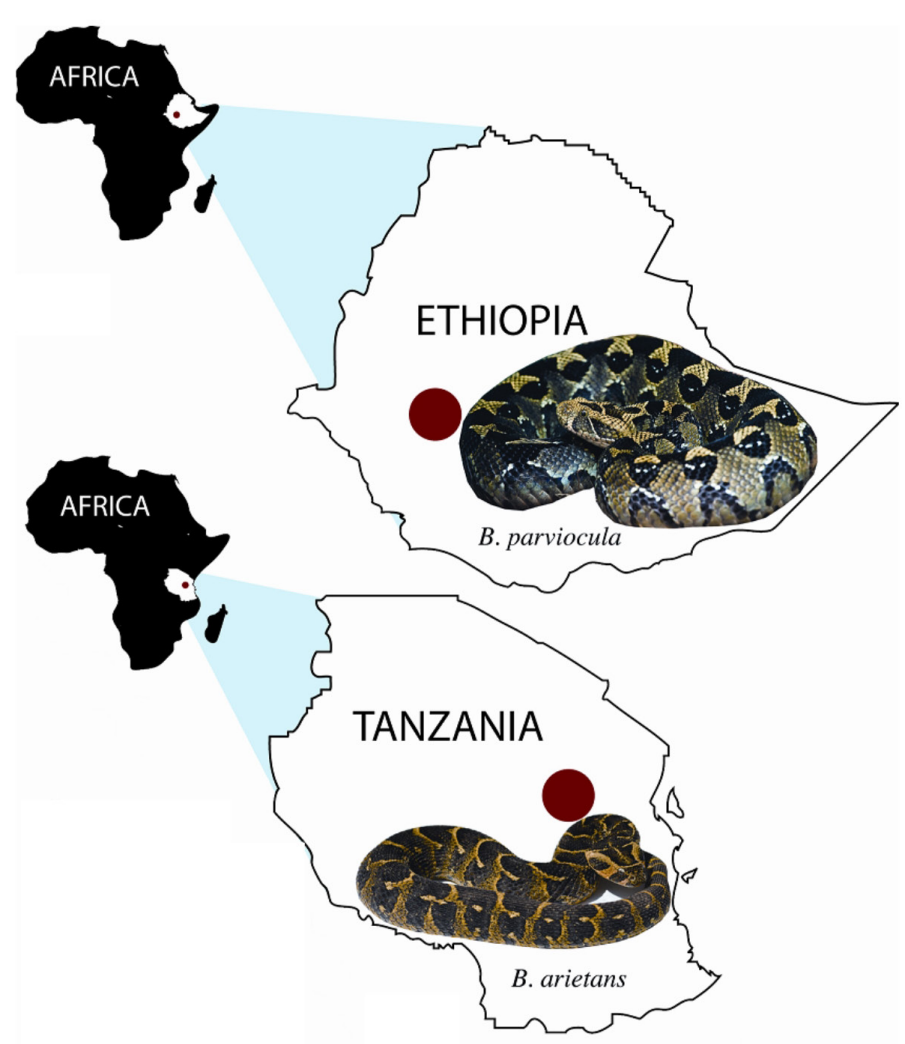

Fig. 1 - Top Map: Collection location of B. parviocula (8 $8^{\circ} 27^{\prime}$ S $1.54^{\prime}$ N $36^{\circ} 21^{\prime} 4.99^{\prime}$ E; elevation 1516 meters). Bottom Map: Collection location of B. arietans collected ( $5^{\circ} 02^{\prime}$ 54. $91^{\prime}$ S $37^{\circ} 3.74684$ E; elevation 1194 meters). Dots indicate approximate locality of collected specimens.

Given that the venom profiles vary in $B$. arietans across their range ${ }^{10}$ the $B$. arietans selected for this study originated from a single location. The snakes were held as part of the private collection maintained by Douglas L. Hotle. The snakes were held in Neodesha ${ }^{\circledR}$ enclosures with an ambient temperature of $27^{\circ} \mathrm{C}$. Rodents (Mus musculus and Rattus norvegicus) were offered as food once every seven days. Water was available ad libitum. Both species were wild caught animals and were considered adults.

Venom collection: Venom was extracted by allowing the snake to bite into a sterile disposable beaker covered with para-film. The venom sample was centrifuged $500 \mathrm{~g}$ for $10 \mathrm{~min}$ at $4{ }^{\circ} \mathrm{C}$, filtered through a $0.45 \mu \mathrm{m}$ filter, and frozen at $-80{ }^{\circ} \mathrm{C}$ until lyophilized.

SDS PAGE: A total of $30 \mu \mathrm{g}$ of B. arietans and B. parviocula venoms were run on a $10-20 \%$ Tricine gel at $150 \mathrm{~V}$ for 90 min using a SureXCell system (Invitrogen). The gel was stained with SimplyBlue (Invitrogen) for one hour and distained overnight with 18 megaOhm water. SeeBlue Plus2 markers were used as controls.

Lethality Dose $\left(\mathbf{L D}_{50}\right)$ : Five groups of eight mice for each venom were housed in cages and observed throughout the quarantine period and experiments. The endpoint of lethality of the mice was determined after $48 \mathrm{hr}$. The venom was dissolved in $0.85 \%$ saline at the highest test dose per mouse. Serial dilutions of 2-fold using saline were made to obtain four additional concentrations. All solutions during the experiment were stored at $0{ }^{\circ} \mathrm{C}$ and warmed to $37^{\circ} \mathrm{C}$ just before being injected into mice. The lethal toxicity was determined by injecting $0.2 \mathrm{~mL}$ of venom (containing dosages ranging between 220 to $13.75 \mu \mathrm{g} / \mathrm{mouse}$ ) into the tail veins of 18-20 g female BALB/c mice. The injections were administered using a $1-\mathrm{mL}$ syringe fitted with a 30 -gauge, 0.5 -inch needle. Saline controls were used. The $\mathrm{LD}_{50}$ was calculated by the Spearman-Karber $\operatorname{method}^{15}(\mathrm{n}=3 \pm \mathrm{SD})$.

Antivenom efficacy dose $\left(\mathbf{E D}_{50}\right)$ : Five groups of eight mice were challenged with a mixture of antivenom containing $3 \mathrm{LD}_{50}$ of venom. SAIMR antivenom (Lot \#: TO1946; South African Vaccine Producers) was diluted with sterile $0.85 \%$ saline. A stock venom solution was freshly prepared at $0{ }^{\circ} \mathrm{C}$ prior to use. For each group of mice, a set concentration of venom was mixed with five different antivenom concentrations and incubated at $37{ }^{\circ} \mathrm{C}$ for $30 \mathrm{~min}$. Each mouse was injected into the tail vein with $0.2 \mathrm{~mL}$ of venom/antivenom mixture in which $3 \mathrm{XLD}_{50} \mathrm{~s}$ were administered per mice. The mice were observed for $48 \mathrm{~h}$ and the percent survival and $\mathrm{ED}_{50}$ was calculated by the Spearman-Karber method ${ }^{15}$ $(\mathrm{n}=3 \pm \mathrm{SD})$.

Hemorrhagic assay: Hemorrhagic activity for crude venom and the collected fractions followed the procedure of OMORI-SATOH et al. $(1972)^{11}$. To test for activity, $0.1 \mathrm{~mL}$ of each of the fractions was injected subcutaneously into the backs of depilated New Zealand rabbits (Oryctolagus cuniculus). After $24 \mathrm{~h}$, the rabbits were sacrificed and hemorrhagic spots measured $(\mathrm{mm})$. The minimal hemorrhagic dose (MHD) was defined as the amount of protein $(\mu \mathrm{g})$ that causes a $10 \mathrm{~mm}$ hemorrhagic spot.

Fibrinogenolytic assay: Fibrinogenolytic activity of crude venom was measured using a procedure modified from SALAZAR et al. $(2007)^{12}$. Two hundred microliters of fibrinogen solution $(2.5 \mathrm{mg} / \mathrm{mL})$ and $100 \mu \mathrm{L}$ of crude venoms $(0.03 \mathrm{mg} / \mathrm{mL})$ were incubated together at $37{ }^{\circ} \mathrm{C}$ for $30 \mathrm{~min}, 1,2,4$ and $24 \mathrm{~h}$. The samples were run on a $4-12 \%$ Bis-Tris gels under reducing conditions using a SureXCell system (Invitrogen). The gels were stained with SimplyBlue (Invitrogen) for one hour and distained with 18 megaOhm water overnight.

Gelatinase assay: Gelatinase activity of the venom fractions was tested using a method modified from HUANG \& PÉREZ (1980) 7 . Fifty microliters of each venom dilution and fractions were placed on a Kodak $\mathrm{X}-\mathrm{OMAT}^{\mathrm{TM}}$ scientific imaging film having a gelatin coating. Hydrolysis of gelatin on the X-ray film was determined by washing the film with tap water following incubation at $37^{\circ} \mathrm{C}$ for four hours in a moist incubator. A resulting clear spot on the $\mathrm{X}$-ray film indicated positive activity. The assay was repeated three times.

Sonoclot Assay: Crude venoms of B. parviocula and B. arietans were tested for their abilities to affect activated clotting time (ACT), clotting rate $(\mathrm{CR})$ and platelet function $(\mathrm{PF})$ on whole human blood according to the method of SÁNCHEZ et al. $(2010)^{13}$.

\section{RESULTS}

SDS PAGE: Pooled venoms of both $B$. parviocula and B. arietans were compared by SDS gel electrophoresis and 10 visible proteins bands were apparent between $\sim 104$ and $8 \mathrm{kDa}$ for $B$. arietans venom while $B$. parviocula had 13 visible bands in the range 128 and $11 \mathrm{kDa}$ (Fig. 2). 


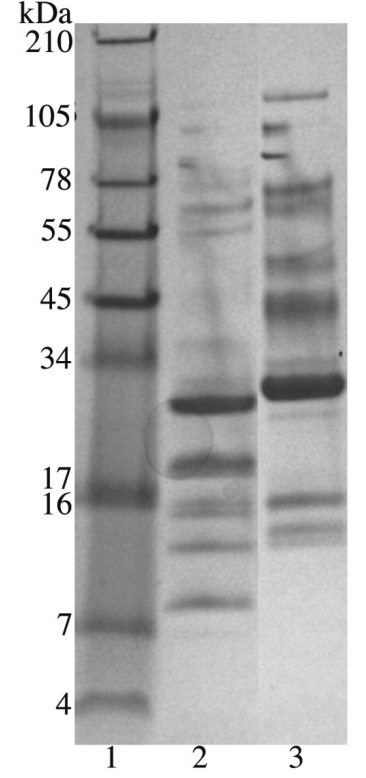

Fig. 2 - A SDS gel electrophoresis of B. arietans and B. parviocula venoms. A total of 30 $\mu \mathrm{g}$ of protein of venoms was run on a 10-20\% Tricine gel under non-reducing conditions at 150 V for 90 min using a SureXCell system. Lanes: 1) SeeBlue Plus2 markers; 2) B. arietans 3) B. parviocula .

Lethality Dose $\left(\mathbf{L D}_{50}\right)$ : The $\mathrm{LD}_{50} \mathrm{~s}$ of $B$. parviocula and $B$. arietans venoms were 1.6 and $1.4 \mathrm{mg} / \mathrm{kg}$ body weight, respectively (Table 1 ).

Antivenom efficacy dose $\left(\mathbf{E D}_{\mathbf{5 0}}\right)$ : The $\mathrm{ED}_{50} \mathrm{~s}$ of SAIMR antivenom on $B$. parviocula and $B$. arietans were $1 / 18.2$ and $1 / 66.7$, in which $1 \mathrm{~mL}$ of SAIMR antivenom incubated with 18.2 and $66.7 \mathrm{mg}$ of venom, respectively can protect $50 \%$ of the population (Table 1 ).

Hemorrhagic assay: The hemorrhagic activities (MHDs) of $B$. parviocula and $B$. arietans were 0.88 and $1.7 \mu \mathrm{g}$, respectively. Western diamondback rattlesnake (Crotalus atrox) venom was used as a control giving a MHD of $2.5 \mu \mathrm{g}$ (Table 1).

Fibrinogenolytic assay: Bitis arietans was able to completely degrade the $\alpha$ chain of human fibrinogen by $30 \mathrm{~min}$ while $B$. parviocula took four hours to completely degrade the $\alpha$ chain (Table 1). However, $B$. parviocula venom was able to degrade the $\beta$ chain much faster (four hours) than $B$. arietans $(24 \mathrm{~h})$. The venom of Crotalus atrox was used as a control and had activity similar to $B$. parviocula venom in the fibrinogenolytic assay. Neither venom affected the $\gamma$ chain (Fig. 3).

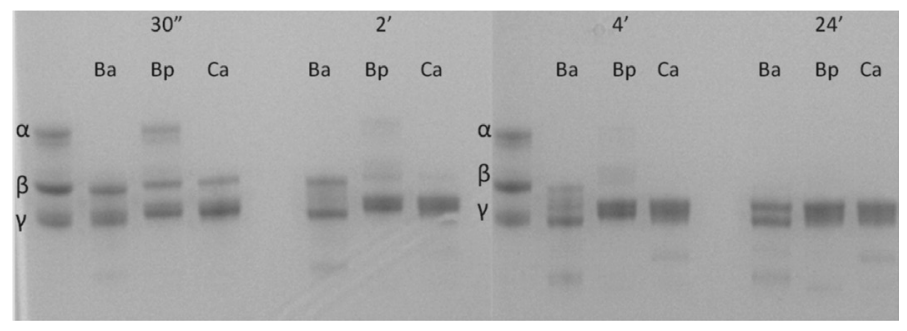

Fig. 3 - Fibrinogenolytic activity of B. arietans $(\mathrm{Ba})$, B. parviocula $(\mathrm{Bp})$ and Crotalus atrox (Ca) venoms. Two hundred microliters of fibrinogen $(2.5 \mathrm{mg} / \mathrm{mL})$ was incubated with $100 \mu \mathrm{L}$ of venom sample $(0.03 \mathrm{mg} / \mathrm{mL})$ and incubated at $30 \mathrm{~min}, 2,4$ and $24 \mathrm{~h}$. The samples were run on a $4-12 \%$ Bis-Tris gel under reducing conditions using a SureXCell system (Invitrogen). The gel was stained with SimplyBlue for $1 \mathrm{~h}$ and distained in 18 mega ohm water overnight.

Gelatinase assay: Bitis parviocula venom did not exhibit gelatinase activity, while B. arietans had a MGD of $6.9 \mu \mathrm{g}$ (Table 1).

Sonoclot assay: The ACTs and CRs were slightly delayed for the venoms of B. arietans and B. parviocula as compared to the normal blood control (Table 1, Fig. 4), while the PFs for both Bitis ssp. venoms were significantly affected, meaning that these venoms had a strong effect on blood platelets.

Table 1

Proteolytic and lethal activities of Bitis arietans and B. parviocula venoms

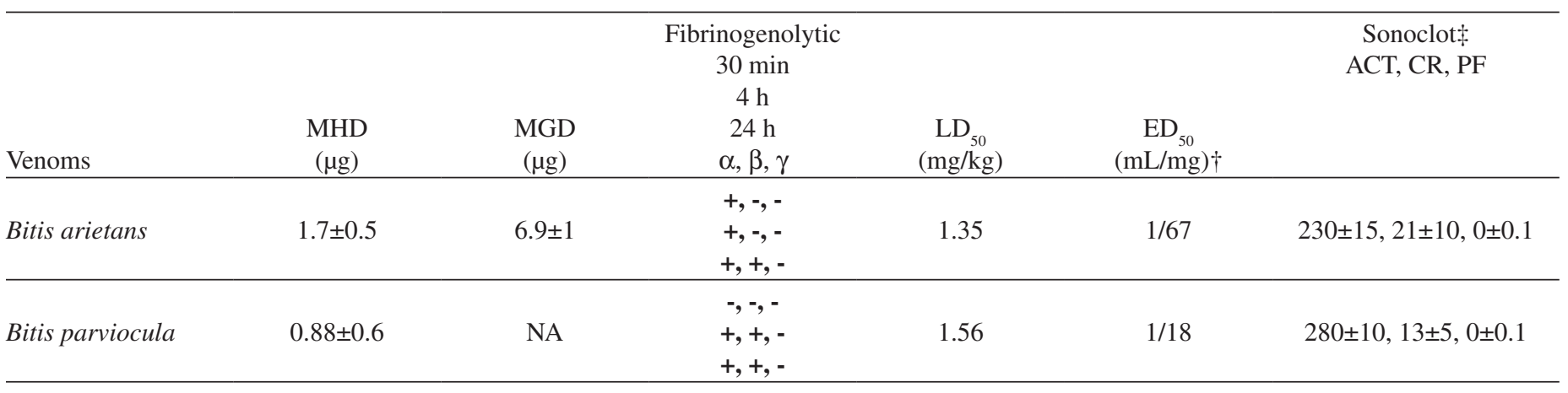

MHD: The minimal hemorrhagic dose is the minimal amount of venom that will cause a $10 \mathrm{~mm}$ hemorrhagic spot according to the method of OMORI-SATOH et al. (1972) ${ }^{11}$. MGD: The minimal gelatinase dose is the minimal amount of venom that will cause a clearing area on an X-ray film according to the method of HUANG \& PEREZ (1980) ${ }^{7}$. NA: No activity. LD $_{50}$ : The lethal dose 50 is the amount of venom that will kill $50 \%$ of a population according to the method of SÁNCHEZ et al. (2010) ${ }^{13}$. $\mathbf{E D}_{50}$ : The effective dose 50 is the titer of anitvenom to venom that will protect $50 \%$ of the population according to the method of SÁNCHEZ et al. (2010) ${ }^{13}$. Antivenom-SAIMR Polyvalent Snake Antivenom. South African Vaccine Producers (PTY) LTD. 1 Modderfontien Rd. Edenvale, Gauteng Lot \# TO1946. †: mL/mg: $1 \mathrm{~mL}$ of antivenom incubated with venom $(\mathrm{mg})$ protects $50 \%$ of the population. +: degradation, -: no degradation. Human fibrinogen was used according to the method of SALAZAR et al. $(2007)^{12}$. \$ACT: activated clot time (s); CR: clot rate (clot signals/min); PF: platelet function. Normal blood control ACT: $189 \pm 15$, CR: $30 \pm 5$, and PF: 2.0 \pm 1.5. A Sonoclot ${ }^{\circledR}$ Coagulation \& Platelet Function Analyzer was used (SIENCO ${ }^{\circledR}$, Inc., Arvada, CO). The method of SÁNCHEZ et al., (2010) ${ }^{13}$ was followed. 


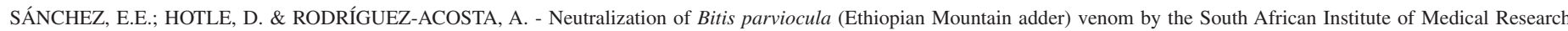
(SAIMR) antivenom. Rev. Inst. Med. Trop. Sao Paulo, 53(4): 213-7, 2011.

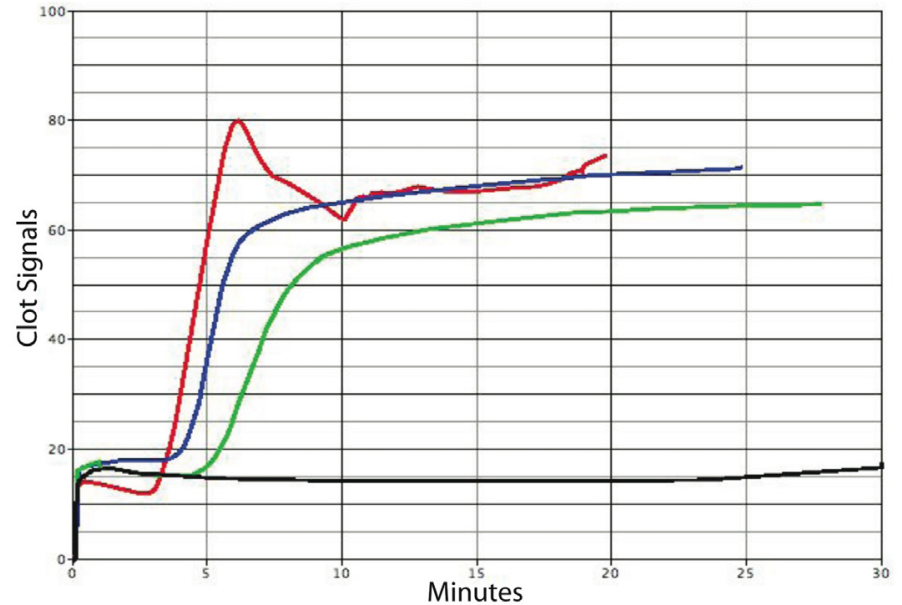

Fig. 4 - The effects of activated clot time (ACT), clot rate (CR) and platelet function (PF) by Bitis venoms. A total of $10 \mu \mathrm{L}$ of venom sample $(3 \mathrm{mg} / \mathrm{mL})$ was added to glass bead activated cuvettes (gbACT+ KIT) containing $350 \mu \mathrm{L}$ of $10 \%$ citrated whole human blood. The ACT, CR and PF were analyzed on a Sienco Sonoclot ${ }^{\circledR}$ Analyzer System. Red: normal blood control, blue: B. arietans venom + blood, green: B. parviocula venom + blood, and black: $C$. atrox venom + blood.

\section{DISCUSSION}

Bitis parviocula has a close taxonomic relationship with other Macrocerastes such as the Puff adder (Bitis arietans), Gaboon vipers (Bitis gabonica and B. rhinoceros), Rhinoceros viper (Bitis nasicornis) among others ${ }^{1}$; and therefore, the presumption was that antivenoms traditionally used for other Bitis would have cross-reactivity against envenomation from $B$. parviocula ${ }^{8,14}$. However, cross-reactivity does not always equal cross-protection ${ }^{3}$. In many cases usage of antivenoms against untested species can be dangerous, or at the least non-beneficial ${ }^{8}$. The addition of the $B$. parviocula into many private and zoological collections increases the likelihood of accidental envenomations by this species. Emergency physicians and snakebite consultants are increasingly faced with treating venomous snakebites inflicted by non-native snakes ${ }^{10}$. An understanding of the cross-protection of specific antivenoms is crucial in the treatment of these emergencies.

Prior to antivenom neutralization, the venoms of B. parviocula and $B$. arietans were compared by SDS electrophoresis in which there were differences in the quantity of proteins in that B. parviocula had three more proteins bands than $B$. arietans (Fig. 2). The venoms were also compared with a series of biological assays.

These venoms were analyzed using a Sonoclot ${ }^{\circledR}$ Coagulation $\&$ Platelet Function Analyzer, in which the measurements are based on the detection of viscoelastic changes of whole blood or plasma ${ }^{6}$. The Sonoclot ${ }^{\circledR}$ provides qualitative (Sonoclot Signature graph) and quantitative (ACT, CR and PF) results on the entire hemostasis process. The activated clotting time (ACT) is the time in which fibrin formation begins, the clotting rate (CR) is the kinetic measurement of fibrin formation and clot development, which is the maximum slope of the Sonoclot Signature during initial fibrin polymerization and clot development, and platelet function (PF) is obtained from the timing and quality of the clot retraction. The values for PF range from 0-5, where 0 represents no clot retraction. A PF higher than 1 represents normal clot retraction and varies from patient to patient. A normal PF contains a sharp peak in the Sonoclot Signature after fibrin formation, as seen on the control sample in Figure 4. The ACTs and CRs were slightly delayed for the venoms of B. arietans and B. parviocula as compared to the normal blood control (Table 1, Fig. 4), while the PFs for both Bitis venoms were significantly affected, meaning that these venoms had a strong effect on blood platelets. Whether there are components in these venoms that bind to the receptors (e.g. $\alpha_{\mathrm{IIb}} \beta_{3}$ ) of platelets thus inhibiting platelet aggregation, or if the venom components degrade the receptors or the platelets themselves is yet to be determined. Crotalus atrox venom, used as a control, rendered the blood sample unclottable (Fig. 4).

The ability to degrade human fibrinogen was also tested. Bitis arietans was able to completely degrade the $\alpha$ chain of human fibrinogen by 30 min while $B$. parviocula took 4 h to completely degrade the $\alpha$ chain (Table 1; Fig. 3). However, B. parviocula venom was able to degrade the $\beta$ chain much faster $(4 \mathrm{~h})$ than $B$. arietans $(24 \mathrm{~h})$. The venom of Crotalus atrox was used as a control and had activity similar to B. parviocula venom in the fibrinogenolytic assay. Neither venom affected the $\gamma$ chain.

Both Bitis venoms were very hemorrhagic with minimal hemorrhagic doses (MHDs) for B. arietans and B. parviocula venoms of 1.7 and $0.87 \mu \mathrm{g}$, respectively (Table 1 ), signifying $B$. parviocula to be twice as hemorraghic. The hemorrhagic activity of $B$. arietans is comparable to the hemorrhagic activity of $C$. atrox and $C$. oreganus helleri venoms $(\sim 2.5-2.3 \mu \mathrm{g})$, while that of $B$. parviocula venom hemorrhagic activity is comparable to the venoms of Crotalus viridis $(\sim 0.7 \mu \mathrm{g})^{13}$. In contrast, B. parviocula did not contain gelatinase activity when tested on an X-ray film, while $B$. arietans had a minimal gelatinase dose (MGD) of $6.9 \mu \mathrm{g}$ (Table 1). These results could imply the absence or low abundance of collagenases in the venom of $B$. parviocula.

The $\mathrm{LD}_{50}$ for $B$. arietans and B. parviocula venoms were 1.35 and $1.56 \mathrm{mg} / \mathrm{kg}$, respectively, and the SAIMR antivenom was capable of neutralizing both venoms quite well (Table 1 ). The $\mathrm{ED}_{50}$ for the SAIMR antivenom against $B$. arietans was $1 / 67$, which translates to $66 \mathrm{mg}$ of venom incubated with $1 \mathrm{~mL}$ of antivenom can protect $50 \%$ of the $\mathrm{BALB} / \mathrm{c}$ mouse population. The $\mathrm{ED}_{50}$ with $B$. parviocula was $1 / 18$, thus the SAIMR antivenom neutralizes $\sim 4$ times more venom of $B$. arietans, which is not surprising since this venom is used in the production of this antivenom (Table 1). Even though SAIMR only protected $50 \%$ of the population with less $B$. parviocula venom, it still protected significantly considering that this venom was not used for the manufacture of the antivenom. According to MALLOW et al. $(2003)^{9}$, the average $B$. arietans venom yield ranges between 100-350 mg, with a maximum of $750 \mathrm{mg}$, while the average venom yield for $B$. parviocula is $100 \mathrm{mg}$ (personal communication by Doug Hotle). Therefore, theoretically, two to three $10 \mathrm{~mL}$ vials of SAIMR antivenom can neutralize the maximum amount of venom from a single extraction of either snake. It is apparent that there exist components in $B$. parviocula venom that are not as easily neutralized as those of $B$. arietans. It is well documented that variation in venom composition exists among Bitis species².

Nonetheless, in this study we have shown that the South African polyvalent antivenom SAIMR did produce paraspecific neutralization of lethality with B. parviocula venom in vivo, and should be considered in emergency treatment. 
SÁNCHEZ, E.E.; HOTLE, D. \& RODRÍGUEZ-ACOSTA, A. - Neutralization of Bitis parviocula (Ethiopian Mountain adder) venom by the South African Institute of Medical Research (SAIMR) antivenom. Rev. Inst. Med. Trop. Sao Paulo, 53(4): 213-7, 2011.

\section{RESUMO}

\section{Neutralização do veneno de Bitis parviocula (serpente da Montanha da Etiópia) pelo antiveneno do Instituto Africano de Pesquisa Médica (SAIMR)}

Background: Serpente das Montanhas da Etiópia (Bitis parviocula) é um viperídeo conhecido somente em poucas localizações do sudoeste da Etiópia. Métodos: Um total de $30 \mu \mathrm{g}$ de veneno de $B$. arietans e B. parviocula foram corridos em gel de 10 a $20 \%$ de tricina. Para se estabelecer a quinquagésima dose de letalidade $\left(\mathrm{LD}_{50}\right)$ foram usados cinco grupos de oito camundongos para cada veneno. A atividade hemorrágica para o veneno cru foi testada. A atividade fibrogenolítica do veneno cru foi medida usando $2,5 \mathrm{mg} / \mathrm{mL}$ de solução de fibrinogênio e $0,03 \mathrm{mg} / \mathrm{mL}$ de veneno cru. A atividade de gelatinase do veneno foi testada em um filme KODAK X-OMAT ${ }^{\mathrm{TM}}$. Venenos crus de B. parviocula e B. arietans foram testados no que diz respeito à sua capacidade de afetar o tempo de coagulação, a velocidade de coagulação e a função plaquetogênica em sangue humano total. Resultado: o antiveneno SAIMR foi confirmado neste estudo no que diz respeito à neutralização da atividade letal do veneno de Bitis parviocula. $\mathrm{ED}_{50} \mathrm{~s}$ do antiveneno SAIMR sobre a $B$. parviocula e B. arietans neutralizou metade de 18,2 e $66,7 \mathrm{mg}$ respectivamente do veneno. As atividades hemorrágicas (MHDs) de $B$. parviocula e $B$. arietans foram respectivamente 0,88 e 1,7 $\mu \mathrm{g}$. Os venenos de $B$. arietans e $B$. parviocula degradaram cadeias $\alpha$ e $\beta$ em tempos diferentes. A cadeia $\gamma$ permaneceu não afetada. $\mathrm{O}$ veneno da $B$. parviocula não mostrou atividade de gelatinase, enquanto o de $B$. arietans teve um MGD de $6,9 \mu \mathrm{g}$. A nível de $3 \mathrm{mg} / \mathrm{mL}$ os venenos crus de $B$. parviocula e $B$. arietans não afetaram significantemente o tempo e a velocidade de coagulação. Conclusões: O antiveneno SAIMR é bastante efetivo para neutralizar o veneno da B. parviocula e deveria ser considerado para o tratamento de envenenamentos por estas serpentes.

\section{ACKNOWLEDGEMENTS}

Financial support was provided by the NIH/NCRR viper resource grant (\#5 P40 RR018300-07) and Texas A\&M University-Kingsville at Texas A\&M University-Kingsville, Kingsville, TX. Thanks to Al Coritz for donating the crude B. parviocula venom, Justin Bennet for his technical assistance, and the faculty and staff at the National Natural Toxins Research Center.

\section{REFERENCES}

1. Böhme W. Eine neue Art der Gattung Bitis (Serpentes,Viperidae) aus Äthiopien. Monit Zool Ital. 1977;(Suppl 9):59-68.
2. Calvete JJ, Escolano J, Sanz L. Snake venomics of Bitis species reveals large intragenus venom toxin composition variation: application to taxonomy of congeneric taxa. J Proteome Res. 2007;6:2732-45.

3. Chippaux JP. Snake venoms and envenomations. Malabar: Krieger; 2006.

4. Currier RB, Harrison RA, Rowley PD, Laing GD, Wagstaff SC. Intra-specific variation in venom of the African Puff Adder (Bitis arietans): differential expression and activity of snake venom metalloproteinases (SVMPs). Toxicon. 2010;55:864-73.

5. Fernández MC, González A. Ethiopian Mountain viper envenomation in South Texas. In: North American Congress of Clinical Toxicology Annual Meeting, September 21-26, 2009, San Antonio, Texas. p. 712. (Clin Toxicol. 2009;47(7).

6. Ganter MT, Hofer CK. Coagulation monitoring: current techniques and clinical use of viscoelastic point-of-care coagulation devices. Anesth Analg. 2008;106:1366-75.

7. Huang SY, Perez JC. Comparative study on hemorrhagic and proteolytic activities of snake venoms. Toxicon. 1980;18:421-6.

8. Keyler D. Venomous snake species commonly displayed in zoos for which specific antivenoms are not available. Association of Zoos and Aquarium's Antivenom Index. Available from: http://www.aza.org

9. Mallow D, Ludwig D, Nilson G. True vipers: natural history and toxinology of Old World vipers. Malabar: Krieger; 2003.

10. Minton SA. Bites by non-native venomous snakes in the United States. Wilderness Environ Med. 1996;7:297-303.

11. Omori-Satoh T, Sadahiro S, Ohsaka A, Murata R. Purification and characterization of an antihemorrhagic factor in the serum of Trimeresurus flavoviridis, a crotalid. Biochim Biophys Acta. 1972;285:414-26.

12. Salazar AM, Rodríguez-Acosta A, Girón ME, Aguilar I, Guerrero B. A comparative analysis of the clotting and fibrinolytic activities of the snake venom (Bothrops atrox) from different geographical areas in Venezuela. Thromb Res. 2007;120:95-104.

13. Sánchez EE, Lucena SE, Reyes S, Soto JG, Cantu E, Lopez-Johnston JC, et al. Cloning, expression, and hemostatic activities of a disintegrin, r-mojastin 1, from the mohave rattlesnake (Crotalus scutulatus scutulatus). Thromb Res. 2010;126:e211-9.

14. Spawls S, Branch B. The dangerous snakes of Africa. Sanibel Island, FL: Ralph Curtis; 1998.

15. Spearman C, Karber G. Alternative methods of analysis for quantal responses. In: Finney DJ, editor. Statistical methods in biological assays. London: Charles Griffin; 1978. p. $1-78$.

Received: 22 May 2011

Accepted: 10 June 2011 